\title{
On the grounding of spin effects in theory of synchrotron radiation
}

\author{
V.A. Bordovitsyn, A.N. Myagkii \\ Tomsk State University, 634050 Tomsk, Russian Federation
}

\begin{abstract}
The problem of the uniqueness in the introduction of spin operators in the synchrotron radiation (SR) theory is discussed. For this purpose we give the invariant spin projections on the basis of the spin projections in the rest frame. The spin equations are used to construct the integrals of motion in the presence of the external electromagnetic field.
\end{abstract}

PACS 03.65.Sq - Semiclassical theories and applications.

Key words: synchrotron radiation, quantum corrections, electron spin operators, spin invariants, spin equations.

${ }^{*}$ Corresponding author. E-mail: bord@urania.tomsk.su 


\section{Introduction}

Synchrotron radiation (SR) is an unique instrument in the research of individual properties of the relativistic electrons [1]. In 1983 the first quantum spin-orientationdependent correction to the synchrotron radiation power was experimentally identified at the Institute of Nuclear Physics, Siberian Branch of the Academy of Sciences (Novosibirsk) [2, 3]. In this experiment, synchrotron radiation was for the first time observed to be dependent on the spin orientation of a free electron moving in a macroscopic magnetic field. The procedure for experimental observation of spin dependence of SR power was based on the Sokolov-Ternov radiative self-polarization effect of the electrons.

To describe the spin effects in relativistic quantum theory one uses spin operators. The spin effects observed in the experiment are determined by eigenvalues of the spin operators. There are different methods of introduction of the spin operators (see Ref. [1]). In this case the situation can arise when the same projections of various spin operators lead to different physical outcomes, such as time of the radiative self-polarization of the relativistic electrons [4]. Hence, the procedure of the unambiguity in the introduction of the spin operators has come into importance. This brings up the question: What spin operator is true? This question is discussed in the paper on the basic of the invariant correlations of the spin projection operators. The paper is devoted to the memory of I.M. Ternov, who was initiator of studies in this field.

\section{Spin operators of the Dirac particles}

In Refs [5, 6] it was shown that all spin effects of SR can be obtained using wave function $\varphi$ of a free electron with certain momentum and arbitrary spin orientation. That is why we shall confine ourselves to the considering of spin operators of Dirac particles with certain momentum $p^{\mu}$ or, in terms of dimensionless momentum, $b^{\mu}=(\gamma, \boldsymbol{b})=p^{\mu} / m_{0} c$.

The simplest method of obtaining the spin operators is to separate from the spin matrices $\gamma^{\mu} \gamma^{5}$ and $\sigma^{\mu \nu}$ the space-like parts (see in Ref. [7]). Then we obtain

$$
\begin{aligned}
\hat{\pi}^{\mu} & =\left(\gamma^{\mu}+i b^{\mu}\right) \gamma^{5}=\left((\boldsymbol{b} \boldsymbol{\sigma}), \rho_{3} \boldsymbol{\sigma}+\rho_{1} \boldsymbol{b}\right), \\
\hat{\Pi}^{\mu \nu} & =\sigma^{\mu \nu}-\left(\gamma^{\mu} b^{\nu}-\gamma^{\nu} b^{\mu}\right)=\left(\rho_{3}[\boldsymbol{\sigma} \boldsymbol{b}], \boldsymbol{\sigma}+\rho_{2}[\boldsymbol{\sigma} \boldsymbol{b}]\right) .
\end{aligned}
$$

Spin operators satisfy commutative correlations for the generators of little Lorentz group.

It can be shown that the mean values of the operators $\hat{\pi}^{\mu}$ and $\hat{\Pi}^{\mu \nu}$ coincide with the classical four-vector of spin $\pi^{\mu}=\left(\pi^{0}, \boldsymbol{\pi}\right)$ and tensor of spin $\Pi^{\mu \nu}=(\boldsymbol{\Phi}, \boldsymbol{\Pi})$.

The eigenvalues of the spin operators in the laboratory frame differ from the eigenvalues $\zeta= \pm 1$ in the rest frame. For example,

$$
\hat{\pi}_{z} \varphi=\zeta \sqrt{1+b_{z}^{2}} \varphi, \hat{\Pi}_{z} \varphi=\zeta \sqrt{1+b_{\perp}^{2}} \varphi
$$

where $b_{\perp}^{2}=b_{x}^{2}+b_{y}^{2}$. In addition, in the laboratory frame the operators $\hat{\pi}^{0}$ and $\hat{\boldsymbol{\Phi}}$ will induce new eigenvalues $\hat{\pi}^{0} \varphi=\zeta b \varphi, \hat{\Phi}_{z} \varphi=\zeta b_{\perp} \varphi$ (see Ref.[9]), which vanish in the

\footnotetext{
${ }^{1}$ We use a metric $g^{\mu \nu}=\operatorname{diag}(-1,1,1,1)$ and 4 -by-4 $\gamma$-matrices $\gamma^{0}=i \rho_{3}, \gamma^{1}=i \rho_{3} \alpha_{1}, \ldots, \gamma^{5}=$ $-i \rho_{1}, \sigma^{12}=\sigma_{3}, \sigma^{10}=-i \alpha_{1}, \ldots$, where $\rho_{i}$ and $\boldsymbol{\alpha}=\rho_{1} \boldsymbol{\sigma}$ are the well known Dirac matrices.
} 
laboratory frame. However, one can introduce such operator

$$
\hat{\boldsymbol{\Sigma}}_{\sigma}=\boldsymbol{\sigma}+\left(1-\rho_{3}\right) \frac{i[\boldsymbol{\alpha} \boldsymbol{b}]}{\gamma+1}
$$

which in the laboratory frame will save the spin eigenvalues in the rest frame, i.e.

$$
(\boldsymbol{\sigma} \boldsymbol{\nu}) \varphi=\left(\hat{\boldsymbol{\Sigma}}_{\sigma} \boldsymbol{\nu}\right) \varphi=\zeta \varphi
$$

where $\boldsymbol{\nu}=(\sin \vartheta \cos \varphi, \sin \vartheta \sin \varphi, \cos \vartheta)$ is the unit vector.

This operator is obtained as a result of unitary transformation of the spin matrices $\sigma$ (see Ref. [1]). Similarly, one can build the spin operator

$$
\hat{\boldsymbol{\Sigma}}_{\rho_{3} \sigma}=\rho_{3} \boldsymbol{\sigma}+\rho_{1} \frac{\boldsymbol{b}}{\gamma}-\frac{\rho_{3} \boldsymbol{b}(\boldsymbol{\sigma} \boldsymbol{b})}{\gamma(\gamma+1)} .
$$

It can be shown that spin operators $\hat{\boldsymbol{\Sigma}}$ possess commutative properties of Pauli matrices $\mathrm{P}$.

In order to transform the eigenvalues of $\hat{\boldsymbol{\Sigma}}$ into the laboratory frame the operator itself must be subjected to the Lorentz transformations (see Ref. [1]).

\section{Invariant properties of spin operators}

The spin operators $\hat{\boldsymbol{\Sigma}}, \hat{\Pi}^{\mu \nu}$, and $\hat{\pi}^{\mu}$ satisfy the invariant relations

$$
\hat{\Sigma}^{2}=\frac{1}{2} \hat{\Pi}_{\mu \nu} \hat{\Pi}^{\mu \nu}=\hat{\pi}_{\mu} \hat{\pi}^{\mu}=3 .
$$

All the spin operators commutate with Hamiltonian of the free Dirac particle with definite momentum $\hat{\gamma}=(\boldsymbol{\alpha} \boldsymbol{b})+\rho_{3}$. This allows to define completely a wave function of particle with given momentum and concrete spin orientation. But due to the noncommutativity of the spin operator projections it is possible to measure simultaneously only the eigenvalues for the absolute value and one of the spin projections. It is known, however, (see, for example, in Ref. [8]) that the same projection of the spin operators, for example $\hat{\Sigma}_{z}, \hat{\pi}_{z}$ or $\hat{\Pi}_{z}$, gives the various eigenvalues ${ }^{3}$. The question arises: What projections of

\footnotetext{
${ }^{2}$ One can meet other operators possessing same properties (see in Ref. [1]). These are Stech spin operator

$$
\hat{\boldsymbol{\Sigma}}_{S}=\rho_{3} \boldsymbol{\sigma}+\left(1-\rho_{3}\right) \frac{\boldsymbol{b}(\boldsymbol{\sigma} \boldsymbol{b})}{\gamma^{2}-1},
$$
}

Foldy-Wouthuysen spin operator

$$
\hat{\boldsymbol{\Sigma}}_{F-W}=\boldsymbol{\sigma}-i \frac{\rho_{3}[\boldsymbol{\alpha} \boldsymbol{b}]}{\gamma}-\frac{[\boldsymbol{b}[\boldsymbol{\sigma} \boldsymbol{b}]]}{\gamma(\gamma+1)}
$$

and so on. However, these operators coincide when applied to solutions of the Dirac equation

$$
\hat{\mathbf{\Sigma}}_{\sigma} \rightarrow \hat{\boldsymbol{\Sigma}}_{\rho_{3} \sigma} \rightarrow \hat{\boldsymbol{\Sigma}}_{S} \rightarrow \hat{\boldsymbol{\Sigma}}_{F-W} \rightarrow \hat{\mathbf{\Sigma}} .
$$

\footnotetext{
${ }^{3}$ For this reason some authors have expressed some doubts concerning the correct application of $\hat{\pi}$ and $\hat{\Pi}$ operators [9].
} 
the spin operators give the correct result? The answer to this question can be found by constructing of relations between the projections of the spin operators.

As a spin projector one can take the antisymmetric tensor $h^{\mu \nu}=(-\boldsymbol{e}, \boldsymbol{h})$ with components $h^{10}=-e_{x}, \ldots, h^{12}=h_{z}, \ldots$. I. In a general case $h^{\mu \nu}$ is not a space-like tensor, therefore the space-like part should be separated out for further application (here, $\rightarrow$ denotes the space-like part of a four-vector or a tensor)

$$
\vec{h}^{\mu \nu}=\left(\gamma^{2}[\boldsymbol{\beta}(\boldsymbol{h}-[\boldsymbol{\beta} \boldsymbol{e}])], \boldsymbol{h}-\gamma^{2}[\boldsymbol{\beta}(\boldsymbol{e}+[\boldsymbol{\beta} \boldsymbol{h}])]\right)=(-\overrightarrow{\boldsymbol{e}}, \overrightarrow{\boldsymbol{h}}) .
$$

Applying the first nonzero invariant we come to the dimensionless space-like tensor (for simplification we put $\boldsymbol{e}=0$ )

$$
\begin{gathered}
S^{\mu \nu}=\vec{h}^{\mu \nu} / \sqrt{I_{1}}=([\boldsymbol{\beta} \boldsymbol{\eta}], \boldsymbol{\eta}-\boldsymbol{\beta}(\boldsymbol{\beta} \boldsymbol{\eta}))=(\boldsymbol{Q}, \boldsymbol{S}), \\
I_{1}=\frac{1}{2} \vec{h}_{\mu \nu} \vec{h}^{\mu \nu}=\gamma^{2}\left[h^{2}-(\boldsymbol{\beta} \boldsymbol{h})^{2}\right], \quad \boldsymbol{\eta}=\frac{\gamma^{2} \boldsymbol{h}}{\sqrt{I_{1}}}=\frac{\gamma \boldsymbol{k}}{\sqrt{1-\beta^{2} \cos ^{2} \alpha}}, \quad(\boldsymbol{\beta} \boldsymbol{k})=\beta \cos \alpha .
\end{gathered}
$$

One can construct the space-like four-vector corresponding to $S^{\mu \nu}$

$$
s^{\alpha}=\frac{1}{2} \varepsilon^{\alpha \beta \mu \nu} S_{\mu \nu} b_{\beta}, \quad S^{\mu \nu}=\varepsilon^{\mu \nu \alpha \beta} s_{\alpha} b_{\beta} .
$$

The components of $s^{\alpha}$ are equal to $s^{\alpha}=((\boldsymbol{\beta} \boldsymbol{\eta}), \boldsymbol{\eta}) / \gamma=\left(s^{0}, \boldsymbol{s}\right)$. According to Eq. (6) they are associated with $S^{\mu \nu}$ by the relations

$$
\boldsymbol{s}=\frac{1}{\gamma} \boldsymbol{S}+\gamma \boldsymbol{\beta}(\boldsymbol{\beta} \boldsymbol{S}), \quad \boldsymbol{S}=\gamma[\boldsymbol{s}-\boldsymbol{\beta}(\boldsymbol{\beta} \boldsymbol{s})]
$$

In the rest frame the spin projectors $\boldsymbol{s}$ and $\boldsymbol{S}$ transform into the unit vector

$$
\boldsymbol{\nu}=\frac{1}{\gamma}\left[\boldsymbol{\eta}-\frac{\gamma}{\gamma+1} \boldsymbol{\beta}(\boldsymbol{\beta} \boldsymbol{\eta})\right]
$$

associated with $\boldsymbol{s}$ and $\boldsymbol{S}$ by Lorentz transformation.

One can show that the spin operators $\hat{\Pi}^{\mu \nu}$ and $\hat{\pi}^{\mu}$ satisfy the relations similar to Eqs (6) and (7). On this basis one can obtain the following invariant relations

$$
\begin{gathered}
s_{\alpha} \hat{\pi}^{\alpha}=\frac{1}{2} S_{\mu \nu} \hat{\Pi}^{\mu \nu}=i n v, \\
(\boldsymbol{\nu} \hat{\Sigma})=\frac{1}{\gamma}(\boldsymbol{S} \hat{\boldsymbol{\pi}})=\frac{1}{\gamma}(s \hat{\boldsymbol{\Pi}}) .
\end{gathered}
$$

Note that the spin operators $\hat{\Sigma}, \hat{\pi}^{\mu}, \hat{\Pi}^{\mu \nu}$ in these relations can be replaced by their classical analogue $\boldsymbol{\zeta}, \pi^{\mu}, \Pi^{\mu \nu}$.

\footnotetext{
${ }^{4}$ In the presence of the external electromagnetic field this tensor can be identified with the tensor $H^{\mu \nu}=(-\mathbf{E}, \mathbf{H})$ (see, for example, in Ref. [10]).
} 
We consider two extreme cases: 1) $\boldsymbol{\beta}\|\boldsymbol{\eta}\| \boldsymbol{k}$, when $\boldsymbol{\beta} \| Z$ and $\beta_{\perp}=0$, 2) $\boldsymbol{\beta} \perp \boldsymbol{\eta} \| \boldsymbol{k}$, when $\boldsymbol{\beta} \perp Z$ and $\beta_{z}=0$. In both cases $\boldsymbol{\nu}=\boldsymbol{k}$ (spin is directed along the $Z$-axis in the rest frame), however, the spin components are different in the laboratory frame

$$
\begin{aligned}
& (\boldsymbol{k} \hat{\Sigma})=\frac{1}{\gamma}(\boldsymbol{k} \hat{\boldsymbol{\pi}})=(\boldsymbol{k} \hat{\boldsymbol{\Pi}}), \text { for } \boldsymbol{\beta} \| \boldsymbol{k}, \\
& (\boldsymbol{k} \hat{\Sigma})=(\boldsymbol{k} \hat{\boldsymbol{\pi}})=\frac{1}{\gamma}(\boldsymbol{k} \hat{\boldsymbol{\Pi}}), \text { for } \boldsymbol{\beta} \perp \boldsymbol{k} .
\end{aligned}
$$

In terms of the eigenvalues of the spin operators these relations coincide with $\zeta$.

In a similar way one can consider the kinematic spin invariants which are induced by the particle motion

$$
\begin{gathered}
(\boldsymbol{\sigma} \boldsymbol{\beta})=(\hat{\boldsymbol{\Sigma}} \boldsymbol{\beta})=\frac{1}{\gamma}(\boldsymbol{\beta} \hat{\boldsymbol{\pi}})=(\boldsymbol{\beta} \hat{\boldsymbol{\Pi}}), \\
(\boldsymbol{\nu} \hat{\boldsymbol{\Phi}})=(s \hat{\boldsymbol{\Phi}})=\frac{1}{\gamma}(\boldsymbol{S} \hat{\boldsymbol{\Pi}})=\frac{1}{\gamma}[\boldsymbol{\eta} \hat{\boldsymbol{\Phi}}] .
\end{gathered}
$$

The first invariants characterize the spin projection onto the direction of motion and the second invariants do the spin projection onto the direction of $[\boldsymbol{k} \boldsymbol{\beta}]$.

One can show that in the BMT approximation (see Ref. [1]) the spin invariant of Eq.(8) remains valid in the presence of the external electromagnetic field also (see Ref. [13]). In particular, in the constant and uniform magnetic field when $\boldsymbol{h}\|\boldsymbol{H}\| \boldsymbol{k}$, the spin projection onto the orbital radius is characterized by the spin operator $\hat{\Phi}_{z}$.

\section{Spin invariants as integrals of motion}

In the presence of the external electromagnetic field the spin operators, generally speaking, stop being integrals of motion. It is known, for instance, that in the constant and uniform electromagnetic fields they satisfy the operator equations similar to the BMT equations [11]. Only some components of spin operators remain the integrals of motion (see also Ref. [12]). Nevertheless one can show [13] that the spin invariants of Eq. (8) remain the integrals of motion if we choose the external electromagnetic field tensor $H^{\mu \nu}$ as a projector $h^{\mu \nu}$. Moreover, the spin invariants allow also to find such direction, for which the spin projection of the moving particle will be always give the same eigenvalue (the precession axis). Such a problem for electron moving in the magnetic field of arbitrary configuration was for the first time solved in Ref. [14], where it was shown that the value of $(\boldsymbol{\nu} \boldsymbol{\Sigma})=$ const, if the unit vector $\boldsymbol{\nu}(\boldsymbol{\beta}, \boldsymbol{H})$ satisfies the equation of BMT type. It is a rather obvious result, as the vector $\boldsymbol{\nu}$ rotates with respect to azimuth with the frequency of the spin precession. We show that this problem can be solved for projectors $s^{\mu}$ and $S^{\mu \nu}$ too. As an example we take the tensor $S^{\mu \nu}$. At the beginning we represent the BMT equation in the form [1]

$$
\frac{d \Pi^{\mu \nu}}{d \tau}=\frac{e g}{2 m_{0} c} \widetilde{H}^{[\mu \rho} \Pi_{\rho}^{\nu]},
$$

where

$$
\widetilde{H}^{\mu \rho}=H^{\mu \rho}+\frac{1}{c^{2}} v^{[\mu} v_{\alpha} H^{\alpha \rho]}+\frac{2 m_{0}}{e g c} v^{[\mu} w^{\rho]}
$$


is an effective electromagnetic field, $v^{\mu}$ and $w^{\mu}$ are the four-velocity and acceleration, $\tau$ is the proper time, the square brackets mean the antisymmetrization with respect to the corresponding indexes. In the case of constant and uniform fields

$$
\frac{d \widetilde{H}^{\mu \nu}}{d \tau}=\frac{g-2}{g c^{2}} H^{[\mu \alpha} w_{\alpha} v^{\nu]} \neq 0,
$$

but

$$
\frac{d}{d \tau}\left(\widetilde{H}_{\mu \nu} \Pi^{\mu \nu}\right)=0
$$

that is rather obvious as $\widetilde{H}_{\mu \nu} \Pi^{\mu \nu}=H_{\mu \nu} \Pi^{\mu \nu}$. Taking into account $S^{\mu \nu}=\overrightarrow{\widetilde{H}}^{\mu \nu} / \sqrt{I_{1}}$ one can find the such moving axes onto which the spin projection will be constant in time.

\section{References}

[1] V.A.Bordovitsyn (ed.), Synchrotron Radiation Theory and Its Development. In memory of I.M.Ternov (World Scientific, 1999, Singapore).

[2] V.N.Korchuganov, G.N.Kulipanov, N.A.Mezentsev et al. Preprint INP 77-83 (Novosibirsk, Institute of Nuclear Physics, 1977).

[3] S.A.Belomesthnykh, A.E.Bondar, M.N.Egorychev et al. Nucl. Instr.and Meth. 227 (1984) 173.

[4] V.G.Bagrov et al. Docl. Akad. Nauk SSSR, 221 (1975) 312.

[5] V.A.Bordovitsyn, I.M.Ternov and V.G.Bagrov, Physics-Uspekhi 38 (1995) 1037.

[6] V.A.Bordovitsyn, V.S.Gushchina and I.M.Ternov, Nucl. Inctr. and Meth. A359 (1995) 34.

[7] V.A.Bordovitsyn and I.M.Ternov, Teor. Mat. Fiz. 51 (1982) 37 (in Russian).

[8] A.A.Sokolov and I.M.Ternov, Synchrotron Radiation (Pergamon Press, New York, 1968; Akademie-Verlag, Berlin, 1968).

[9] V.N.Baier and V.M.Katkov, Yad. Fiz. 3 (1966) 81 (in Russian).

[10] Zhukovskii and N.S.Nikitina, Zh. Exp. Teor. Fiz. 64 (1973) 1169 (in Russian).

[11] I.M.Ternov and V.A.Bordovitsyn. Vestn. Mosk. Univ. III, 21, N 3, (1980) 8, ibid. 23, N 6 (1982) 72 (in Russian).

[12] I.M.Ternov, V.G.Bagrov and V.A.Bordovitsyn, Izv. Vuz. Fiz. 10, N 4, (1967) 41 (in Russian).

[13] V.A.Bordovitsyn and I.M.Ternov, Teor. Mat. Fiz. 59 (1984) 220 (in Russian).

[14] Ya.S.Derbenev, A.M.Kondratenko and A.N.Skrinskii, Sov.Phys.-JETP 33 (1971) 658. 